\title{
Regional and neuraxial anesthesia for visceral organ transplant- status quo at german transplant centers
}

\author{
C. Massoth 1 , M. Lange ${ }^{1}$, A. Zarbock 1 , O. Djuren², M. Wenk ${ }^{1}$
}

${ }^{1}$ Department of Anesthesiology, Intensive Care and Pain Medicine, University Hospital of Muenster - Muenster (Germany), ${ }^{2}$ Department of Anesthesiology, Intensive Care and Pain Medicine, University Hospital of Oldenburg -

Oldenburg (Germany)

\section{Background}

The use of perioperative epidural anesthesia leads to a reduction of morbidity and potentially influences mortality. However, the risk-benefit ratio of regional and particularly neuraxial anesthesia for visceral organ transplant surgery is controversial due to the beneficial effects on pain and organ function on the one hand and risk of bleeding and infection and their respective sequelae on the other hand. So far, only results from case series and small-scale clinical trials suggest feasibility and effectiveness of epidural anesthesia (EDA) for both renal and liver transplant.

\section{Objective}

To determine the status quo of the use of regional anesthesia for visceral organ transplant in Germany

\section{Methods}

Ethics Committee approval: (2015-689-f-S)

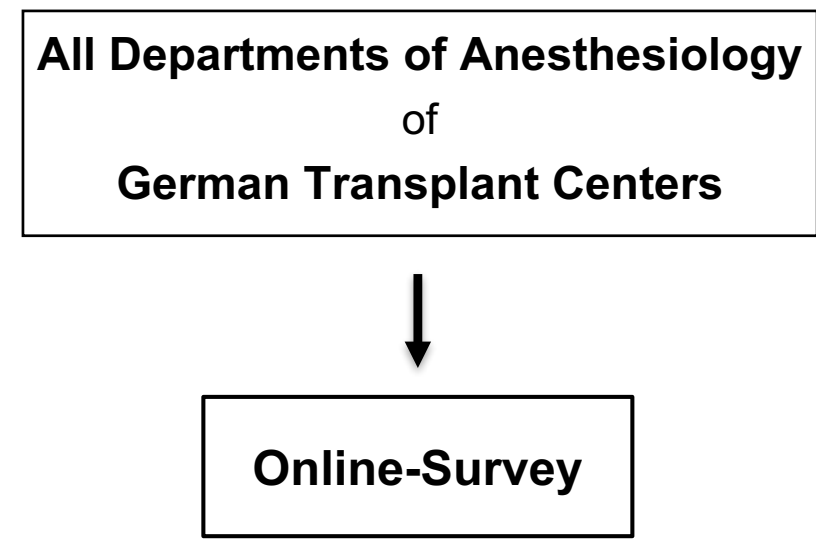

use of EDA/regional anesthesia for visceral organ transplantation in 2015

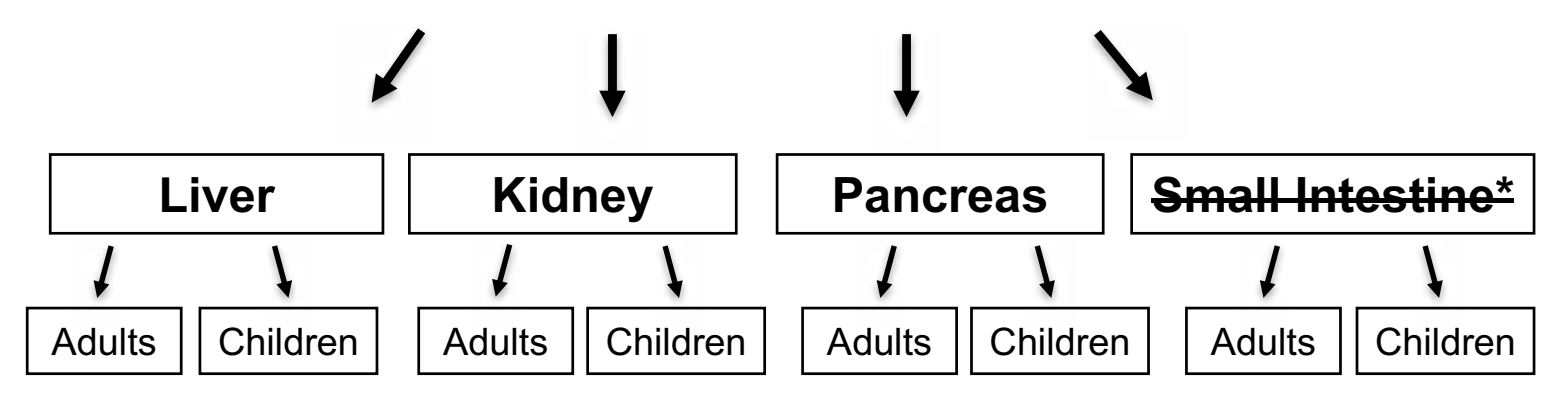

\section{Results}

total reply rate: $83 \%$

$34 / 41$ transplant centers (TC) completed the survey

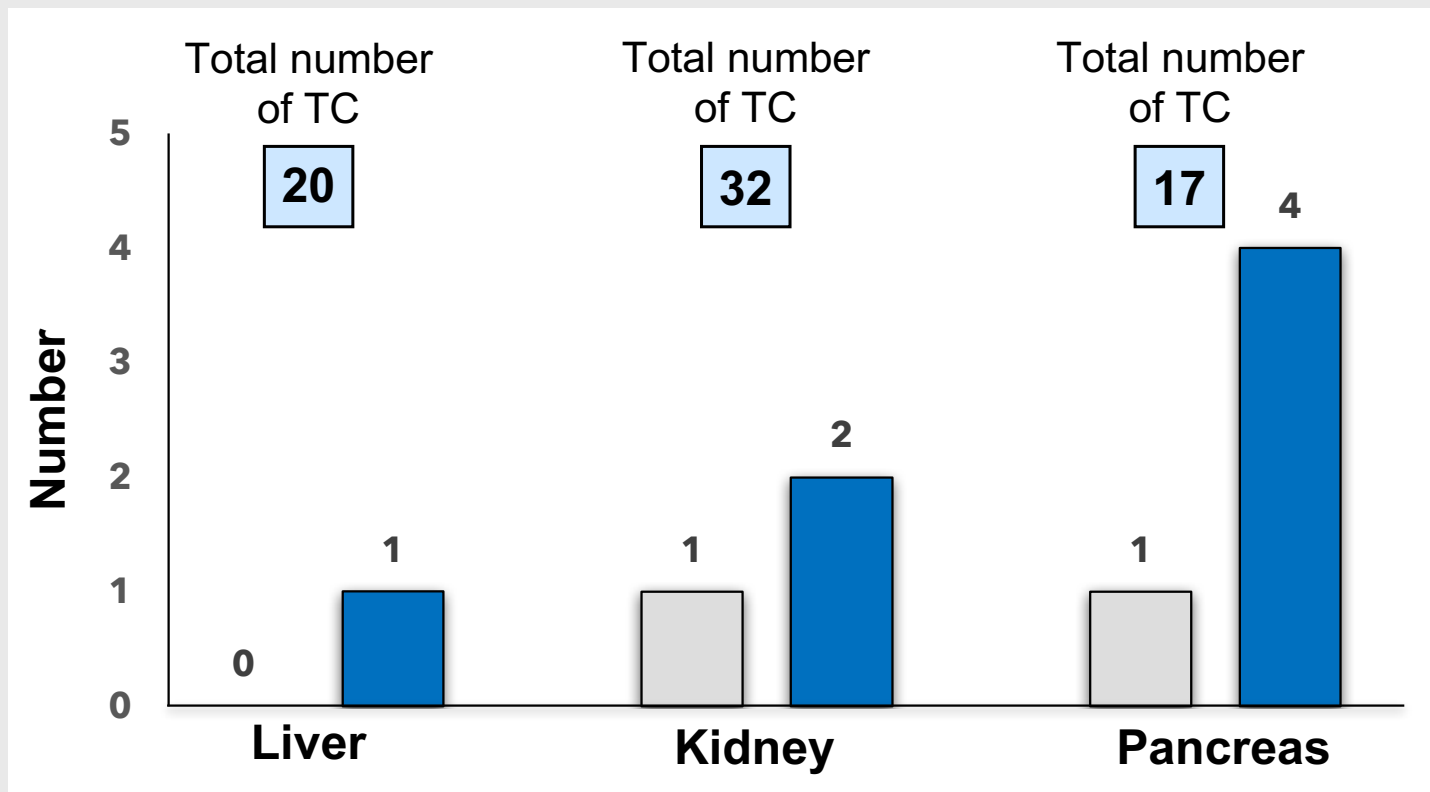

-most frequently mentioned reasons against EDA:

-a high risk of bleeding during liver transplantation

-a significant but clinically not relevant pain reduction for renal transplant patients

-a high risk of infection in pancreas transplantation

-regional anesthesia techniques other than EDA were used even less frequently and only in renal transplant patients

Figure: The number of centers using EDA for graft recipients as a standard procedure (grey) or based on individual decision making (blue)

use of EDA in children <16y

-Liver recipients: 1/7 TC based on individual decision making

-Kidney recipients: 1/5 TC as standard procedure

-Pancreas recipients: 0/1 TC

-in organ donors, EDA use was also restricted because of an increased risk of bleeding, particularly during partial liver donation

\section{Conclusion}

The lack of evidence-based information on the risk-benefit ratio of regional anesthesia for visceral organ transplantation is reflected in the cautious use of these techniques in German transplant centers 